Cell Research, (2001); 11(1):74-80

\title{
Rice bicoid-related cDNA sequence and its expression during early embryogenesis
}

\author{
YANG ZHi XING ${ }^{1}$; Guo Yong AN ${ }^{2}$, Zhi PING ZHU ${ }^{1, *}$ \\ ${ }^{1}$ Shanghai Institute of Plant Physiology, Chinese Academy of Sciences, Shanghai 200032, China \\ ${ }^{2}$ Department of Biology, Henan University, Kaifeng 475001, China
}

\begin{abstract}
Bicoid is one of the important Drosophila maternal genes involved in the control of embryo polarity and larvae segmentation. To clone and characterize the rice bicoid-related genes, one cDNA clone, Rb24 (EMBL accession number: AJ2771380), was isolated by screening of rice unmature seed cDNA library. Sequence analysis indicates that $\mathrm{Rb} 24$ contains a putative amino acid sequence, which is homologous to unique 8 amino acids sequence within Drosophila bicoid homeodomain (50\% identity, $75 \%$ similarity) and involves a lys-9 in putative helix 3. Northern blot analysis of rice RNA has shown that this sequence is expressed in a tissue-specific manner. The transcript was detected strongly in young panicles, but less in young leaves and roots. This results are further confirmed with paraffin section in situ hybridization. The signal is intensive in rice globular embryo and located at the apical tip of the embryo, then, along with the development of embryo, the signal is getting reduced and transfers into both sides of embryo. The existence of bicoidrelated sequence in rice embryo and the similarity of polar distribution of bicoid and Rb24 mRNA in early embryo development may implicates a conserved maternal regulation mechanism of body axis presents in Drosophila and in rice.
\end{abstract}

Key words: Rice bicoid-related DNA, polar distribution, embryogenesis.

\section{INTRODUCTION}

The establishment of embryonic polarity is one of the critical events in embryogenesis. The polar distribution of maternal mRNA can be traced to the unfertilized egg cell. After fertilization, its translational products trigger the zygotic target genes which define the embryonic region and further direct the pattern formation and body plan during embryogenesis. In Drosophila, Bicoid is one of the important maternal genes involved in the control of embryo polarity and larvae segmentation. The protein encoded by bicoid is a homeodomaincontaining transcriptional factor, which organizes a groups of polycomb genes expressed in early de-

\footnotetext{
* Corresponding author: 300 Fenglin Road, Shanghai Insti tute of Plant Physiology, Chinese Academy of Sciences, Shanghai 200032, China. E-mail: zhu@iris.sipp.ac.cn

Received Nov-8-2000. Revised Dec-26-2000. Accepted Jan-9-2001.
}

velopment of embryo and therefore named morphogen[1]. In plant, The zygotic embryo in asymmetric organized embryo sac is also highly polarized[2]. However, we still know less about the maternal regulation mechanism of embryo development in plant.

As regard the maternal mRNA and its role in plant embryogenesis, there were already some investigations up to date. Fucus, one of the brown algae, has been used as a model system to study the induction of cellular polarity and embryogenesis. The presence of maternal mRNA in fucus embryo [3],[4] suggests that maternal regulation of embryo development is not unique to animal. Recently, the localization of actin mRNA during the establishment of cell polarity in fucus embryo is further investigated[5]. In higher plants, stored mRNA and 
seed germination have been investigated in various species from leguminous plant[6] to cereal crops [7],[8] using transcriptional and translational inhibitors. The involvement of stored maternal messages in early stages of plant embryo development has been suggested by experiments in which cultured rice and barley florets produced embryos that utilized labeled amino acids for protein synthesis but did not use labeled nucleotides for mRNA synthesis until globular stage[9], [4]. In our revious investigations on Arabidopsis t-DNA insertional embryonic mutant, down regulation of alanyl-tRNA synthetase gene expression in early embryogenesis lead to arrested embryo development in globular stage[10], which indicates that the translation of stored mRNA may be important for early embryo development. Furthermore, a maternal effect mutant, MEDEA, was identified in Arabidopsis and the MEA gene was cloned and characterized[11]. This results suggest that similar maternal regulation mechanism may exist in both animals and higher plants.

To understand if the bicoid-like gene is also present and functioning in higher plant, in present paper, we report a rice bicoid-related sequence isolated from rice cDNA library using a PCR amplified probe from Drosophila bicoid gene and further investigates its polar distribution in early embryogenesis of rice.

\section{MATERIALS AND METHODS}

\section{Plant material}

The round grain rice (Oryza sativa L. subsp. japonica) was grown in phytotron at $25^{\circ} \mathrm{C}$, under illumination for $12 \mathrm{~h}$. The date of anthesis were marked with painting and the samples in different developing stages were collected for further microscopic observation and in situ hybridization.

\section{Probe preparation}

Two probes were PCR amplified and Dig labeled

Probe 1, a fragment of Drosophila bicoid sequence containing part homeodomain and flanking region was PCR amplified from Drosophila oophorom cDNA library using following primers: BCH1(forward): 5' TCTCAAATAGCAGAGCTGGAGC3' , and BCH3 (reversed): 5' CCATTGTA GTTGTAGTAGGCG3' . The amplified $358 \mathrm{bp}$ fragment was cloned into pBluescript, checked with sequence analysis and then Dig labeled using a Dig DNA labeling and detection system (Boehringer Mannheim).

Probe 2, another fragment only containing $147 \mathrm{bp}$ bicoid homeodomain region was amplified with primer $\mathrm{BCH} 1$ (forward)
5'TCTCAAATAGCAGAGCTG GAGC3' and BCH2 (reversed) 5' ATTGGATCTTGTGACGACGCC3' . This fragment was Dig labeled and used for second round library screening.

\section{Screening of cDNA library}

A rice unmature seed cDNA library was screened with the Diglabeled probe1. Hybridization was performed in $5 \times \mathrm{SSC}, 1 \%$ Blocking reagent, $0.1 \% \mathrm{~N}$-lauroylsarcosine, $0.1 \% \mathrm{SDS}$ at $60^{\circ} \mathrm{C}$ for $16 \mathrm{~h}$ according to the instruction of Boehringer Mannheim kit mentioned above. The positive clones selected by probe 1 were further screened by probe 2 at $65^{\circ} \mathrm{C}$ for hybridization.

\section{Sequence analysis}

The positive clones were checked with agarose electrophoresis and Southern blot. Then the insert fragment was cloned into pBluescript $\mathrm{SK}^{+}$for sequence analysis using Applied Biosystem 373A Sequencer.

\section{RNA blot analysis}

Total RNA was prepared separately from different tissues according to the method of Prescot and Martin (1987)[12]. For RNA dot blotting, the different RNA samples were blotted to nitrocellulose membranes using Bio-Dot Microfiltration Apparatus and the blots were hybridized with the Dig-labeled probe at $65^{\circ} \mathrm{C}$ in high stringency.

\section{Preparation of rice embryo paraffin sections}

For paraffin section, wild-type seeds of various developmental stages were fixed in FAA (formalin/glacial acetic acid/70\% ethanol, 5:6:89) for $10-12 \mathrm{~h}$ at $4^{\circ} \mathrm{C}$. Then, followed by dehydration and infiltration, the samples were embedding with paraffin. Samples were sectioned at 5-8 mm and stained with hematoxylin.

\section{In situ hybridization on paraffin sections}

For the synthesis of antisense and sense transcripts of rice bicoid-related $(\mathrm{Rb})$ sequence, $\mathrm{Rb} 24$ coding region without poly (A) tails was subcloned into pBluescript $\mathrm{SK}^{+}$and transcribed in vitro with Dig-UTP by using T3 or T7 polymerase (Boehringer Mannheim). Standard paraffin sections of rice embryo were hybridized with Dig labeled probes according to the procedure of Coen et al.(1990)[13]. Immunological detection of the hybridized probe was performed using Dig-nucleic acid detection system as mentioned above.

\section{RESULTS}

\section{Cloning of rice bicoid related $c D N A$ sequence}

To approach the presence of bicoid related gene in rice, first of all, two probes were PCR amplified using DNA template from Drosophila oophoron cDNA library. The probe 1 is a 358 bp fragment. Sequence analysis indicates that this fragment contains part of Drosophila bicoid homeodomain and part of 3' end flanking region. The probe 2 was further PCR amplified from probe1. This probe 
contains only part of homeodomain region and used for second check when rice cDNA libarary is screened by probe 1 .

Before cDNA libarary screening, Southern blot was performed using restriction enzyme treated rice total DNA and Dig labeled Probe1. The results showed strong hybridized bands in Hind III and EcoR I digested rice total DNA (data not shown). This confirmed the potentiality for further library screening.

For library screening, the rice unmature seed cDNA library was serially diluted and screened by probe1 and then followed by plaque hybridization and checked by probe 2 in high stringency. Four independent clones from screening were cloned into pBluescript $\mathrm{SK}^{+}$and sequenced. Among them, sequence analysis indicates pRb24 is a bicoid-related one.

pRb24 (EMBL accession number: AJ277138) is a $1061 \mathrm{bp}$ cDNA sequence containing a coding region of $555 \mathrm{bp}$ which encodes a 185 amino acid sequence. To compare the homeodomain conserved region between RB24, Drosophila bicoid gene[14], [1] and other chicken and mouse bicoid-related genes[15], Block Maker software were used for analysis of highly conserved regions of proteins. The results indicates two conserved block regions in bicoid (Drosophila), and Rb24(rice), Ptx1(chicken), Gsc(mouse), Otx1(mouse) bicoid-related genes, which just cover putative helix1 and helix3 region in bicoid homeodomain (Fig 1A). Among them, an amino acids sequence of KNRRRHK in helix3, which is unique for Drosophila bicoid homeodomain [1], is significantly homologous to $\mathrm{Rb} 24$ sequence (50\% identity; $75 \%$ similarity) (Fig 1B). In particular, lysine $(\mathrm{K})$ at residue 9 of the predicted third helix, which is responsible for unusual DNA binding specificity of bicoid homeodomain[16] is also present in $\mathrm{Rb} 24$. Comparison of bicoid unique amino acids sequence with those of other bicoid related genes shows a similar homology with Ptx1 (50\% identity), Gsc(50\% identity), and Otx1 (50\% identity), although some other part of these bicoid related genes have higher homology with bicoid. Accordingly the Rb24 is one of the bicoid-related sequence.
Expression of RB24 during rice embryogenesis

To approach the expression and function of $\mathrm{Rb} 24$ in rice, the RNA dot blot analysis was performed using Dig labeled probe1. The results indicate that $\mathrm{Rb} 24$ expression is in a tissue specific manner. Its transcriptional products are mainly accumulated in rice young panicles, while less in leaf and only trace in root samples (data not shown).

To further confirm the expression and function of $\mathrm{Rb} 24$ during embryogenesis, the investigation of rice embryogenesis and in situ hybridization was conducted. During the rice pro-embryogenesis, the embryonic apical-basal polarity is initiated in globular stage (Fig 2A), which can be observed in pearlike embryo, recognizable by differentiation of small apical meristem cells and large basal region cells (Fig 2B, C). The embryo is axis symmetry in these stages. Later, the protrusion of coleoptile from ventral side of pear-like embryo (Fig 2D) marks the end of pro-embryo developmental period. It is also a sign of emergence of dorsal-ventral polarity. After this, the embryo is getting to become asymmetric along with the differentiation of shoot apex, root apex, true leaf primordia and other organs (Fig 2E$\mathrm{H})$. In mature rice embryo, the main organs, such as those mentioned above, are arranged in ventral side of embryo, which is quite different with dicotyledonous plant, for instance, Arabidopsis[10]. On the basis of cytological investigation of rice embryogenesis mentioned above, in situ hybridization experiments were performed to determine the expression pattern of Rb24 sequence. The standard paraffin sections of rice embryo in main developmental stages are first checked with sense RNA probe. There is not any specific signal found in rice embryo sections (Fig 3G-H), which indicates the signal in following experiments should be caused by anti-sense probe.

When the paraffin section of rice embryo in different stages are used for in situ hybridization, strong signal is found in early globular embryo (Fig $3 \mathrm{~A}-\mathrm{B}$; E-F). The intensity of signal is gradually reduced along with the embryo development. In mature embryo, there is only trace signal can be detected in tips of true leaf and shoot apex after longer incubation for color reaction(data not shown). This shows that Rb24 is mainly functioning in early stages of rice embryogenesis. 
PtX1 QRRQRTHFTSQQLQELEATFQRNRYPQMSMREEIAVWT NLTE PRVAVWEKNRRAKWAKRE GSC KRRHRTIFTDEQLEALENLFQETKYPQVGTREQLARKVHLREEKVEVWEKNRRAKWARQK OtX1 QRRERTTFTRSQLDVLEALEAKTRYPQIFMREEVALKINLPESRVQVWFKNRRAKCAQQQ Rb24 EHSKRPSRVWSQA DELVILRGLITYRTSYIRGQLSAKVS-- - TTQLSDKVRRLKQKYQM BCd PRRTRTTFTSSQIAELEQHFLQGRYLTAPRLADLSAKLALGTAQVKIWEKNRRRRHKIQS

$\mathrm{BCd}$

$\mathrm{Rb}$

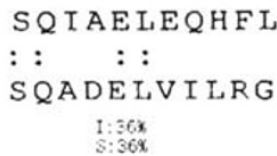

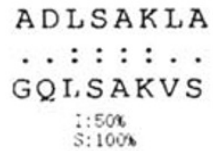

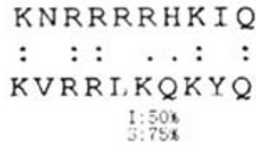

Fig 1. Alignment of bed homedomain with corresponding region of rb and other bicoid-related homeo genes (A) The 60 amino acids homeodomain region of bicoid ( Drosophila) is compared with those of ptx1(chicken), Gsc(mouse), Otx1(mouse) (Lamonerie 1996) and rb24(rice) sequences. Two conserved blocks from BLOCK MARKER analysis are boxed. The predicted a-helixs (Burglin 1993) are indicated on the top of alignment and the residue 9 of helix3 is marked by*. Rb24 contains a 16 aa insertion, which is pointed by an arrow $\uparrow$.

(B) The comparison of helix 1, 2, 3 region between bicoid and rb homeodomain. I: identity; S: similarity

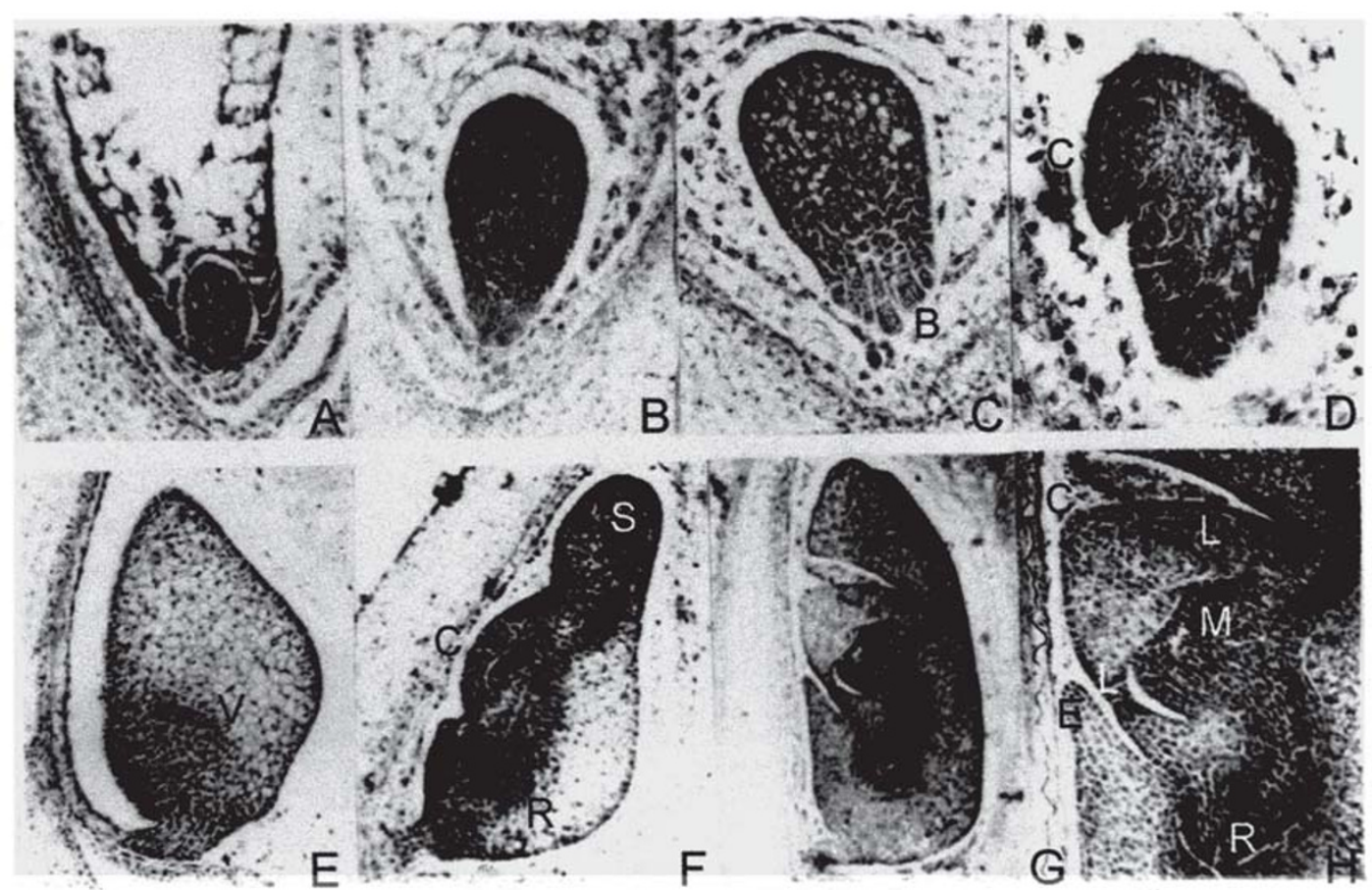

Fig 2 . Developing rice embryo The development of rice embryo involves following stages: A) Globular stage. The embryo is in axis symmetry B) Pear-like embryo stage. The upper part of the embryo contains small meristematic cells. In the meantime, the large vacuolar cells appear in lower part of embryo. C) Late pear-like embryo stage. The base cell (B) appears in lower part of the embryo. D) Coleoptile stage, the protrusion of coleoptile (C) marks the end of pro-embryo period. After this stage, the rice embryo develops into asymmetric form. E) late stage of D). In this stage, the embryo is in triangle shape. The differenciation of vascular tissue(V) can be observed. F) Differentiation embryo. The scutellum(S), primary root(R) and coleoptile(C) already emerged, the dorsal-ventral sides being distinquishable. G) Nearly matured embryo. In this stage, the leaf primodia $(\mathrm{L})$; primary $\operatorname{root}(\mathrm{R})$; shoot apical meristem $(\mathrm{M})$; coleoptile $(\mathrm{C})$ and epiblast $(\mathrm{E})$ are clearly recognizable. H) Enlargement of part of G). A)-E) $320 \times$; F)-G) $128 \times$; H) $410 \times$ times enlargement. 
In globular embryo, the strong signal is located in upper part of embryo (Fig 3A-B; C-D), although the morphological embryonic polarity still can not be clearly observed yet in this stage. This suggests that polar distribution of $\mathrm{Rb} 24$ is involved in polarity formation and establishment of apical-basal axis in early rice embryo.

In pear-like embryo, the signal turns to both dorsal and ventral sides of embryo with reduced intensity (Fig 3C). Furthermore, when coleoptile is protruded from pear-like embryo, the signals are located in the coleoptile protrusion region and dor- sal side of embryo (Fig 3D). This showed a dorsalventral expression pattern of RB24 in pear-like embryo. On the basis of the results mentioned above, it is likely a two phases distribution of $\mathrm{Rb} 24$ is existed in pro-embryogenesis of rice. Firstly, the polar distribution of Rb24 in top of globular embryo directs the establishment of apical-basal axis and formation of a symmetric embryo. Then, the further distribution toward both sides of embryo causes the dorsal and ventral differentiation leading to an asymmetric embryo.

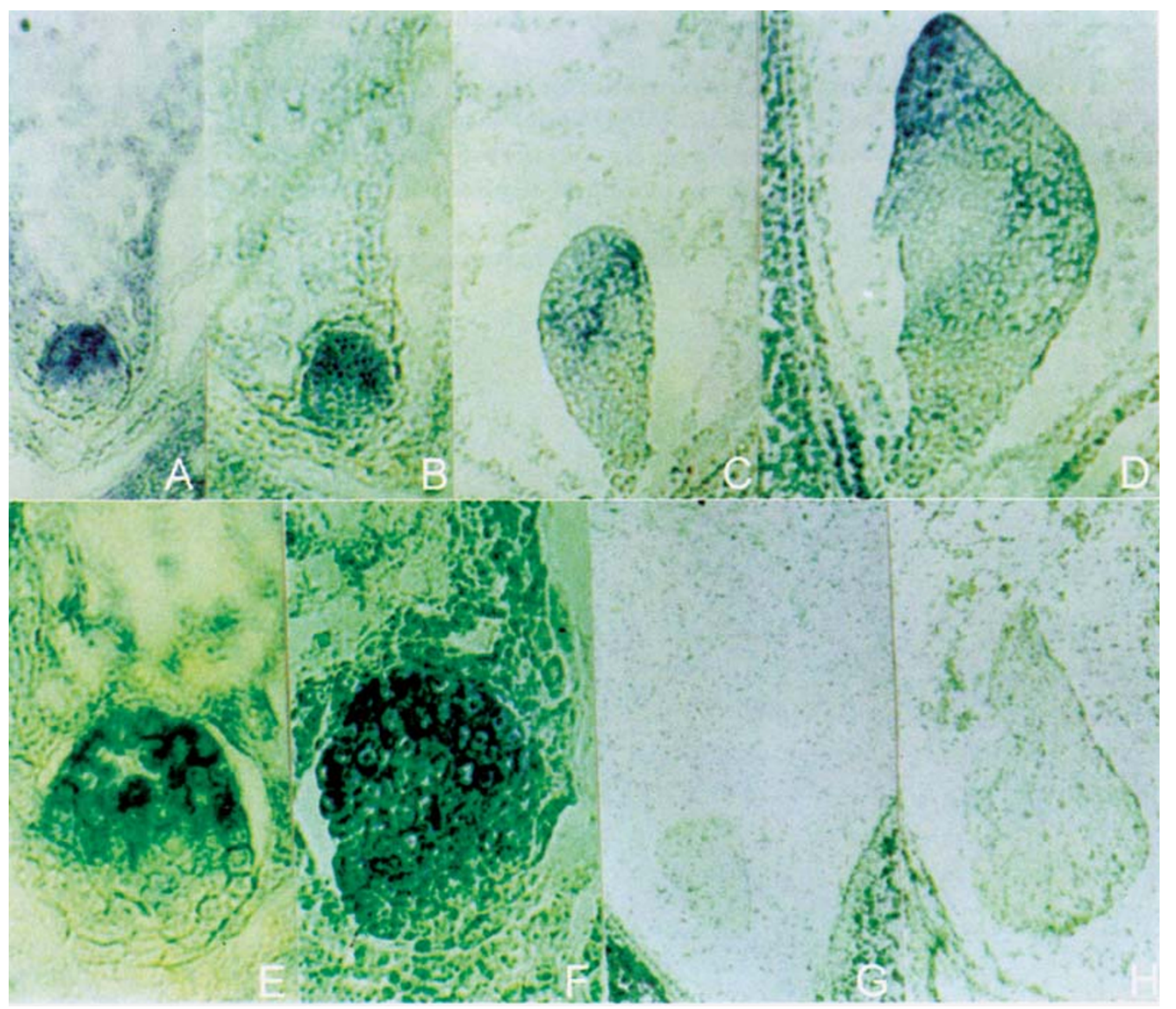

Fig 3. In situ hybridization of Rb24 in developing rice embryo (paraffin sections) Anti-sense Rb24 RNA probe was Dig-labeled and hybridized with longitudinal paraffin sections (A-F) as described in methods. Sense Rb24 RNA probe was used control (GL). The transcript-specific hybridization signal is visualized as blue color. A)-B) globular embryo. The signal is located in the upper part of embryo. C) pear-like embryo; D) late pear-like embryo. The signal is transferred into both sides of embryo along with embryo development. E)-F) enlargement of A), B).

A-B) $600 \times$; C-D) $300 \times$; G-H) $240 \times$; E-F) $1500 \times$ times enlargement

\section{DISCUSSION}

The bicoid gene and its function as morphogen have been extensively investigated in Drosophila [1]. To approach the similar mechanism of mater- nal regulation in embryo development, some bicoidrelated genes are recently reported in chick[15], [17] mouse[18] and other animals, which are mostly characterized by the lys-9 of helix3[16]. However, 
there is still not any bicoid-related gene reported in higher plant yet, although the polarity of embryo has been reported in higher plant(2). The Rb24 is isolated from rice cDNA library using Drosophila bicoid derived probe. By means of computing analysis, a conserved block covers helix 3 of homeodomain in ptx1, Gsc, Otx1, Bicoid and Rb24 sequence is indicated. Among them, the lysine at residue 9 of helix3, a landmark of bicoid-related gene, is also present in Rb24. This showed Rb24 contained a bicoid-related homeodomain. The isolation of Rb24 allowed further observation of its localization in rice embryo development and to compare with Drosophila bicoid. It is clear that embryo developmental course in Drosophila and rice is quite different. However, some similar polar distribution in early embryogenesis is indeed observed in rice. As we know, In Drosophila, the bicoid mRNA is strictly localized to the anterior tip of the embryo. Correspondingly, Bicoid morphogen is distributed in a concentration gradient along the anterior-posterior axis of the embryo[1]. The expression of $\mathrm{Rb} 24$ in rice early embryogenesis is also in similar way too. In globular embryo, Rb24 is located in the apical tip of the embryo (Fig 3A-B; E-F), although along with the embryo development and differentiation of dorsal and ventral structure, Rb24 mRNA is getting to transfer to both sides of embryo (Fig 3C-D).

The polar distribution of Rb24 mRNA is not directly related to organ formation, since not any organ or primordia can be clearly observed in rice globular embryo and pear-like embryo, while the signal is detected in these embryos. It is likely the polar distribution of Rb24 is involved in axis formation. During the early embryogenesis its polar distribution includes two phases. First it is located in the apical tip of embryo and then transfer to both sides of embryo, just concomitant with the establishment of apical-basal and dorsal-ventral axis. If so, the existence of bicoid-related gene in early embryo of different organisms with different organs should not be surprised.

The similarities between mechanisms that govern early embryonic polarity in the nematode Caenorhabditis elegans and in Drosophila has been compared and an ancient molecular mechanism for establishing embryonic polarity based on maternal mRNA localization was put forward[19]. The similarity of polar distribution of bicoid and $\mathrm{Rb} 24$ mRNA in early embryo development may implicates a conserved maternal regulation mechanism of body axis presents in Drosophila and in rice through long time evolution.

\section{ACKNOWLEDGMENTS}

The authors want to thank Wang Z.Y. for providing rice cDNA library; Wu Y.L. (Shanghai Institute of Cell Biology) for Drosophila oophorom cDNA library; Luo D. for valuable suggestion on in situ hybridization.

This paper was financially supported by Chinese National Natural Science Foundation (Project No. 39980002). The Rb24 DNA sequence were deposited to EMBL Nucleotide Sequence Database. The EMBL Accession\#: AJ2771380.

\section{REFERENCES}

[1] Driever W. The Bicoid Morphogen: Concentration-dependent Transcriptional activation of Zygotic Target Genes during Early Drosophila Development. In Transcriptional Regulation (Mcknight S.L. and Yamamoto K.R. Eds.) Cold Spring Harbor Laboratory Press: New York 1992; 1221-50.

[2] West MAL, Harada JJ. Embryogenesis in Higher Plants: An Overview. Plant Cell 1993; 5:1361-9.

[3] Kropf DL et al. Protein synthesis and morphogenesis are not tightly linked during embryogenesis in Fucus. Developmental Biology 1989; 134:452-61.

[4] Castle LA, Meinke DW. Embryo-defective mutants as tools to study essential function and regulatory processes in plant embryo development. Seminars in Developmental Biology 1993; 4:31-9.

[5] Bouget FY, Gerttula S, Shaw SL. Quatrano RS. Localization of Actin mRNA during the Establishment of cell Polarity and Early Cell Divisions in Fucus Embryo. Plant Cell 1996; 8:189-201.

[6] DureL S. (1979) Role of stored messenger RNA in late embryo development and germination. In: the Plant seed: Development, Preservation and Germination (Rubenstein I., Philips R.L., Green C.E. and Gengenbach,B.G. Eds.) Academic Press: New York 1979: 113-27.

[7] Spiegel S, Marcus A. Polyribosome formation in early wheat embryo germination independent of either transcription or polyadenylation. Nature London 1975; 256: 228-30.

[8] Zhu ZP, Wang ML, Shen RJ, Tang XH. On the kinetics of nucleic acid changes and the formation of long-lived mRNA during embryogenesis of rice. In: The Role of RNA in Development and Reproduction (II) (Eds.: Niu MC., Chuang HH.) Science Press: Beijing 1981; 919-30. 
[9] Raghavan V. Embryogenesis in Angiosperms; A developmental and experimental study. Cambridge University Press, New York 1986.

[10] Sun JG, Yao XL and Zhu ZP. An Arabidopsis embryonic lethal mutant with reduced expression of alanyl-tRNA synthetase gene. Cell Research 1998; 8:119-34.

[11] Grossniklaus U, Vielle-Calzada JP, Hoeppner MA, Gagliano WB. Maternal control of embryogenesis by MEDEA, a Polycomb group genes in Arabidopsis. Science 1998; 280:446-50.

[12] Prescott A, Martin C. A rapid method for the quantitative assessment of levels of specific mRNA in plants. Plant Molecular Biology Reporter 1987; 4(4):219-4.

[13] Coen ES, et al. Floricaula: a homeotic gene required for flower development in Antirrhinum Majus. Cell 1990; 63:1311-22.

[14] Berleth T, Burri M, Thoma G, Bopp D, Richstein S, Frigerio G, Noll M, Nusslein-Volhard C. The role of localization of bicoid RNA in organizing the anterior pattern of the Drosophila embryo. EMBO J 1988; 7(6): 1749-56.
[15] Lamonerie T, Tremblay J, Lanctot C, Therrien M, Gauthier Y, Drouin J. Ptx1, a bicoid-related homeo box transcription factor involved in transcription of the proopionmelanocortin gene. Genes and Development 1996; 10:1284-95.

[16] Hanes SD, Brent R. DNA specificity of the bicoid activator protein is determined by homeodomain recognition helix residue 9. Cell 1989; 57:1275-83.

[17] Lanctot C, Lamolet B, Drouin J. The bicoid-related homeoprotein Ptx1 defines the most anterior domain of the embryo and differentiates posterior from anterior lateral mesoderm. Development 1997; 124(14): 2807-17.

[18] Simeone A, Acampora D, Mallamaci A, Stornaiuolo A, D'Apice MR, Nigro V, Boncinelli E. A vertebrate gene related to orthodenticle contains a homeodomain of the bicoid class and demarcates anterior neuroectoderm in the gastrulating mouse embryo. EMBL J. 1993; 12: 2735-47.

[19] Kimble J. An ancient molecular mechanism for establishing embryonic polarity? Science 1994; 266:577-8. 